\title{
Reporting of joint-replacement outcomes lacking
}

A

better understanding of the effects hip-implant materials have on the risk of early replacement surgery will require increased reporting of joint-replacement outcomes across Canada, according to the Canadian Institute for Health Information (CIHI).

In a new study, CIHI found that people with large-diameter metal-on-metal hip replacements are 1.6 times more likely to need early revision surgery than patients with more common metal-on-plastic implants.

Marketed as a solution for younger and more active patients, "the promise was that these implants would wear less because they're metal, and be more stable because they're a bigger construct," says Dr. Michael Dunbar, chair of the Canadian Joint Replacement Registry at CIHI. "It turns out the components are very sensitive to malposition, so being off by a few degrees can create excessive metal debris, which we're finding in some patients causes strange immunological reactions in their tissue."

Hip implants typically work well for $10-15$ years or more, but CIHI's analysis found that patients with metal-on-metal replacements had a $5.6 \%$ risk of revision surgery within five years, compared to a $2.7 \%$ risk for those with metal-on-plastic devices. The study also reveals that patients face an increased risk of early revision surgery if they are under age 55 or have their initial operations in the Atlantic region.

However, it's hard to dig deeper into these trends without comprehensive reporting of operation outcomes across the country, argues Greg Webster, CIHI's director of primary health care information and clinical registries. "At this time, just two jurisdictions [British Columbia and Ontario] mandate submissions to the Canadian Joint Replacement Registry, and voluntary participation doesn't get us the more than $90 \%$ coverage important for this type of registry."

Dunbar estimates that less than half of the available data on hip replacements

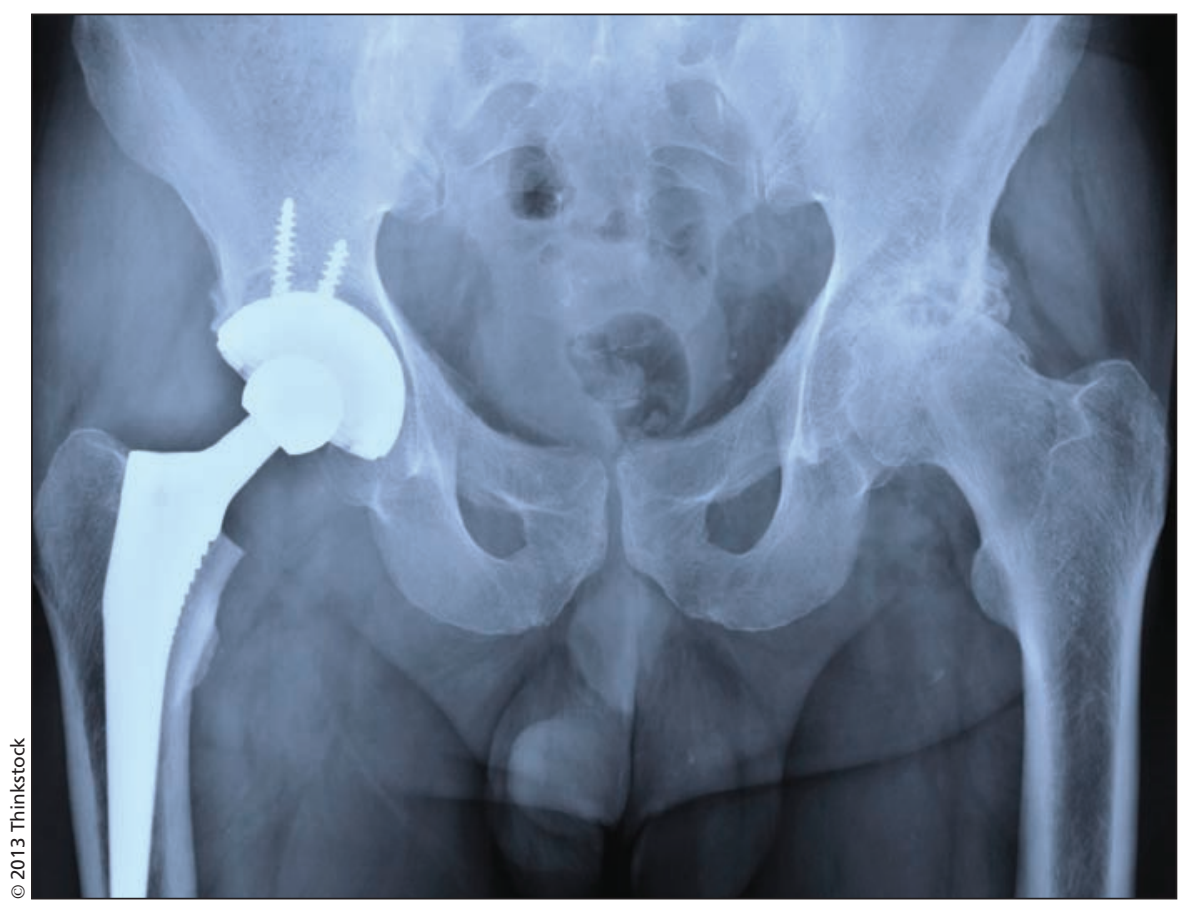

People with large-diameter metal-on-metal hip replacements are 1.6 times more likely to need early revision surgery than patients with more common metal-on-plastic implants, states new study.

are reported to the registry. "I don't understand, as a surgeon, why we're spending so much money on complicated patients, on a national priority, and we're not tracking $100 \%$ to see what happens."

Canadian surgeons are already using metal-on-metal implants more selectively, possibly in response to early alarm bells sounded in countries with more robust reporting. "We suspect, although this has not been tested, that they're looking at international registry findings," says Webster.

A spate of recalls and class action suits has recently prompted regulators to take a second look at the devices as well. Last year, Health Canada issued a safety advisory recommending surgeons monitor all patients with metal-on-metal implants, even if they show no signs of complications. The United States Food and Drug Administration has also voiced concern about the devices, cautioning that "at this time, it is not possible to predict who will experience a reaction, what type of reaction they might have, when the reaction will occur, or how severe the reaction will be."

Surgeries to replace faulty implants represent a "tremendous burden on patients and the health care system," says Webster. "Across Canada, approximately $10 \%$ of all the hip-replacement surgeries each year are revision surgeries, so that's eating up capacity in the system and means people may need to wait longer for their first surgery."

Revision surgeries are also more complex than initial replacements, leading to lengthier hospital stays, longer recovery times and higher procedure costs, according to CIHI. This brings into question the use of implant materials that may hasten replacement surgery.

"The most important thing going forward is to ask "why?" Dunbar argues, "And you can't begin to ask that question unless you have the data." - Lauren Vogel, CMAJ

CMAJ 2013. DOI:10.1503/cmaj.109-4551 